\title{
OPTMIZING HEAT TRANSFERING SURFACE SHAPE OF BRAZED PLATE HEATEXCHANGER
}

\author{
Rostislav Li ${ }^{1}$, Aleksandr Odintsov ${ }^{1}$, Sandra Sidenko ${ }^{1,2}$ \\ ${ }^{1}$ SIA "EKO AIR", Latvia; ${ }^{2}$ Riga Technical University, Latvia \\ ekoair@icloud.com, aleksandr.odintsov@ekoair.lv, sandysid@inbox.lv
}

\begin{abstract}
Brazed plate heat exchangers are widely used in many areas of present industry, which indicates their advantages instead of traditional shell-tube heat exchangers. The using of rational combination of the shape and dimensions of the channels in combination with a correct layout of the heat exchanger allows to achieve a higher heat transfer intensity than in the shell-tube ones. The purpose of this work is to optimize the shape of the heat transfer surfaces of the plates, to improve the thermal properties of the plate heat exchanger and further introduction of it into production. As a base model the plate heat exchanger type 538 was chosen. In this work the surface shape of the plates of the heat exchangers was optimized taking into account the flow characteristics in the channels. Thermal and hydraulic parameters of the base and optimized surface were calculated by using the software package UNICALC Predict. Comparison with the base model calculations has shown that a heat exchanger with an optimized shape has a higher heat transfer coefficient up to $6 \%$ and decreased hydraulic pressure drop up to $9 \%$ as well as a higher heat transfer intensification effectivity coefficient $\approx 9 \%$. Based on the obtained calculation results a prototype of the tool and prototype of the heat exchanger with improved thermal properties were designed and manufactured.
\end{abstract}

Keywords: plate heat exchangers, corrugations, turbulent flow, heat transfer, coefficient of fluid resistance.

\section{Introduction}

Plate heat exchangers have found wide application in many areas of the modern industry, as well as power engineering, heating systems, ventilation, air conditioning, refrigerating machinery and in many others systems.

So wide and various range of scopes of the plate heat exchangers testify to their advantages over traditional shell-tube. First of all, they include: compactness, a high heat-transfer coefficient with a relatively small hydraulic resistance, ease of maintenance, etc [1;2].

SIA "EKO AIR", Salaspils (Latvia), is a manufacturer of brazed plate heat exchangers, which consist of a series of thin metal plates with a corrugated surface, and are fastened among themselves by brazing. Plates are made of sheet material, mainly stainless steel, with a thickness of $0.3 \mathrm{~mm}$ using cold stamping, as a result of which a corrugated surface is formed with an interleaving of ledges and dints. The shape and size of the corrugations are different and depend on the type of the heat transfer plate. Holes on the edges of the plate serve for the pass of the working media in the channels formed by the adjacent plates. The heat exchanger plates are assembled into packages depending on the demanded quantity and are additionally reinforced by the outer reinforcement plates to create a technological system for filling the parallel channels. One liquid or gaseous medium moves through the even channels, the other - through the odd.

The process of heat transferring in plate heat exchangers is complex. In such constructions complex shapes of heat transfer surfaces and channels formed by them are used, in which the heattransferring media flow undergoes artificial turbulization due to the curvilinear shape of the channels and flow brake-off when flowing around the tops of the corrugations of the plates [3].

Note that in plate heat exchangers the turbulent mode is observed already at Reynolds numbers from 50 to 200, which is provided by the geometric shape of the heat transfer surface of the plates [4].

Therefore, the use of a rational combination of the shape and size of the channels for various media with a rational layout allows to achieve the heat transfer intensity 2-3 times greater than in shell-tube heat exchangers with the same values of $R e$ [5].

Therefore, despite of the wide publications [6;7] currently available, the task of optimizing the construction of the plate heat exchangers with improved thermal, physical and hydraulic parameters remains relevant.

The purpose of this work is to optimize the shape of the heat-transferring surfaces of plates, with improved thermal properties, for further manufacturing of the heat exchangers. 


\section{Materials and methods}

As a base model the heat exchanger NB538 type with maximal operating pressure in 31 bars and $\mathrm{H}$-type of the plate has been accepted.

Typical construction of the heat exchanger NB538 consists of a front cover plate (1), rear cover plate (5), and a package of heat transferring plates (2) brazed together in a vacuum environment. The plate assembly forms a series of parallel channels, in which, in counter current mode, a liquid (water) flows participating in heat exchanging. The channels for the heating medium are arranged through one, alternating with the channels for the heated medium. The flow pattern - counter current. This arrangement forms a heat exchange assembly with four manifolds $(Q 1, Q 2, Q 3, Q 4)$ for supplying and discharging the liquid Fig. 1.a. The heat exchanger plates are identical in design. Every second plate in the assembly is rotated by the $180^{\circ}$ with respect to the previous one. Fig. $1 \mathrm{~b}$.

a)

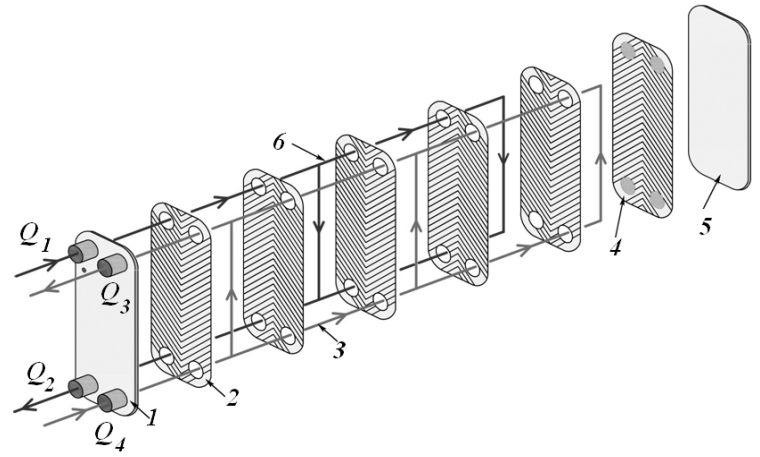

b)

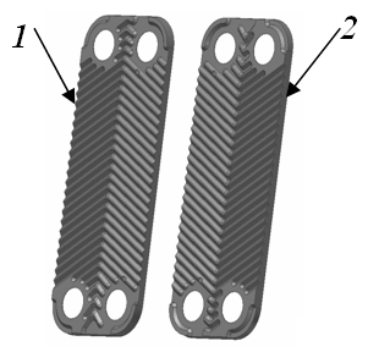

Fig. 1. Flow pattern into channels (a): 1 - front cover plate; 2 - first working plate;

3 - heated media; 4 - last working plate; 5 - back cover plate; 6 - heating media; $Q_{1}, Q_{2}$ - manifolds for inlet and outlet of the heating media; $Q_{3}, Q_{4}$ - manifolds for inlet and outlet of the heated media.

Design of plates(b): 1 - left, 2 - right
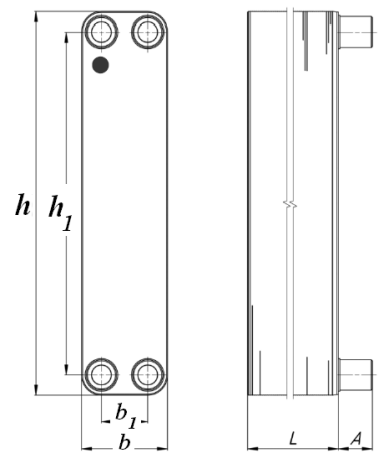

Fig. 2. Geometry of heat exchanger NB5388

Instead of the base version of the NB538H-V0 heat exchanger, for the NB538H-V1-V4 variants, parameters such as Psk (the number of plates) were optimized, respectively thickness $\mathrm{L}$ and weight $\mathrm{M}$ were changed. Thickness $L$ and weight $\mathrm{M}$ were determined: $L=14.83+P s k$ x $2.35 \mathrm{~mm}$, $M=3.024+P s k \times 0.186 \mathrm{~kg}$. The external geometry of the heat exchanger type NB538H is shown in Fig. 2, the sizes of the basic and optimized variants are shown in Table 1.

Adjacent plates with corrugations of the type "fish bone" are forming complex channels for the movement of the working media. In the direction of the flow of the working media a network of expanding and narrowing channels is forming. Such channels are formed by the points of contact of the ledges of the corrugations of adjacent plates and their dints. Flowing around the point of contact of the corrugations, the fluid continuously changes its direction of movement. In addition, the velocity of its movement is constantly changing due to the alternation of sections of expansion and narrowing of the cross section of the channel. It is known that fluid layers moving at a convex surface (along an arc of a smaller radius) have a greater velocity, while that of a concave surface has a lower velocity [8;9]. Changing of the fluid velocity for the working media significantly affects the heat transfer ratio. 
Geometrical characteristics of heat exchangers, $\mathbf{m m}$

\begin{tabular}{|c|c|c|c|c|c|c|c|c|}
\hline \multirow{2}{*}{ Type } & \multicolumn{7}{|c|}{ Dimension } & \multirow{2}{*}{$\begin{array}{c}\text { Mass } \\
(\boldsymbol{M}), \mathbf{k g}\end{array}$} \\
\cline { 2 - 10 } & $h$ & $h_{1}$ & $b$ & $b_{1}$ & $L$ & $A$ & $P s k$ & 7.41 \\
\hline NB538H-V0 & 531 & 471 & 123 & 64 & 83.8 & 27 & 20 & 9.43 \\
\hline NB538H-V1 & 531 & 471 & 123 & 64 & 60.8 & 27 & 30 & 7.41 \\
\hline NB538H-V2 & 531 & 471 & 123 & 64 & 83.8 & 27 & 20 & 7.41 \\
\hline NB538H-V3 & 531 & 471 & 123 & 64 & 106.8 & 27 & 20 & 9.43 \\
\hline NB538H-V4 & 531 & 471 & 123 & 64 & 83.8 & 27 & 30 & 9 \\
\hline
\end{tabular}

Therefore, taking into account the above, such parameters as the angle of opening of the corrugations $\alpha_{1}{ }^{\circ}$, angle of inclination of the corrugations $\alpha_{2}^{\circ}$, plate thickness $t$, step between the corrugations $c_{1}$ and other parameters changed.

Fig. 3 shows the shape of the channels formed by the pair of adjacent plates for a standard heat exchanger type NB538-V0. The geometrical dimensions of the base variant and optimized plates are listed in Tab. 2
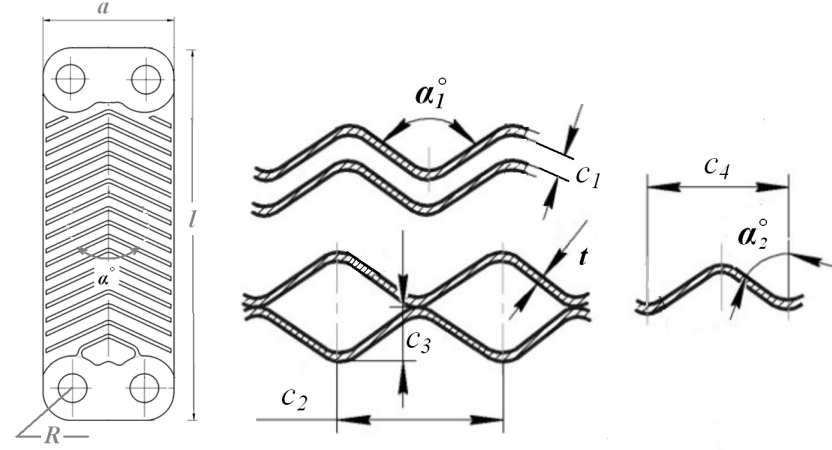

Fig. 3. Geometrical characteristics of plates

Table 2

Geometrical characteristics of plates of heat exchanger NB538H, mm

\begin{tabular}{|c|c|c|c|c|c|c|c|c|c|c|c|c|}
\hline \multirow{2}{*}{ Type } & \multicolumn{11}{|c|}{ Dimension } & \multirow{2}{*}{$\begin{array}{c}\text { Mass } \\
(M), \mathrm{kg}\end{array}$} \\
\hline & $a$ & $l$ & $R$ & $a^{\circ}$ & $\alpha_{I}^{o}$ & $\alpha_{2}^{o}$ & $c_{1}$ & $c_{2}$ & $c_{3}$ & $c_{4}$ & $t$ & \\
\hline V0 & 118.86 & 526.86 & 26.5 & 122 & 136.20 & 42.45 & 1.45 & 12.47 & 2.31 & 6 & 0.39 & 0.167 \\
\hline V1 & 118.80 & 526.80 & 26.5 & 122 & 134.80 & 44.80 & 1.41 & 12.45 & 2.35 & 6 & 0.38 & 0.178 \\
\hline V2 & 118.71 & 526.51 & 26.5 & 122 & 138.56 & 46.80 & 1.75 & 16.56 & 2.35 & 7 & 0.43 & 0.178 \\
\hline V3 & 118.71 & 526.51 & 26.5 & 122 & 136.80 & 43.05 & 1.48 & 12.60 & 2.29 & 6 & 0.43 & 0.178 \\
\hline $\mathrm{V} 4$ & 118.71 & 526.51 & 26.5 & 122 & 140.41 & 49.58 & 1.84 & 16.56 & 2.29 & 7 & 0.43 & 0.178 \\
\hline
\end{tabular}

The operating parameters of the heat exchanger of the basic variant NB538H-V0 with total capacity $100 \mathrm{~kW}$ are presented below. Operating pressure -2 bars, allowed pressure drop $25 \mathrm{kPa}$.

Hot water side: inlet temperature $-70^{\circ} \mathrm{C}$; outlet temperature $-40^{\circ} \mathrm{C}$; Reynolds number -1316 ; mass flow rate $-0.794 \mathrm{~kg} \cdot \mathrm{sec}^{-1}$; volume flow rate $-0.0008 \mathrm{~m}^{3} \cdot \mathrm{sec}^{-1}$.

Cold water side: inlet temperature $-5^{\circ} \mathrm{C}$; outlet temperature $-60{ }^{\circ} \mathrm{C}$; Reynolds number -1056 ; mass flow rate $-0.4422 \mathrm{~kg} \cdot \mathrm{sec}^{-1}$; volume flow rate $-0.0004 \mathrm{~m}^{3} \cdot \mathrm{sec}^{-1}$.

Using the software package UNICALC Predict, a preliminary calculation of the thermophysical and hydraulic parameters of the developed heat transferring plates was carried out. Such parameters as heat transfer coefficients and the coefficient of fluid resistance were determined.

The heat transfer coefficient, when the media moves in the channels formed by corrugated plates, is calculated according to equation (1):

$$
N u=a \cdot \operatorname{Re}^{n} \cdot \operatorname{Pr}^{m}\left(\frac{P r}{P r_{w}}\right)^{0.25},
$$




$$
\begin{gathered}
\operatorname{Pr}=\frac{\mu \cdot C_{p}}{\lambda}, \\
\operatorname{Re}=\frac{\rho \cdot V \cdot S}{\mu},
\end{gathered}
$$

where $\mathrm{Nu}$ - heat transfer coefficient;

$\mathrm{Pr}$ - Prand number for the liquid;

$\operatorname{Pr}_{w}$ - Prand number near the wall;

$C_{P}$ - specific heat capacity at constant pressure, $\mathrm{J} \cdot(\mathrm{kg} \cdot \mathrm{K})^{-1}$;

$\lambda$ - heat transferring coefficient of the media, $\mathrm{W} \cdot(\mathrm{m} \cdot \mathrm{K})^{-1}$;

$\mu$ - dynamic viscosity coefficient, $\mathrm{Pa} \cdot \mathrm{sec}$;

$S$ - area of the cross section, $\mathrm{m}^{2}$;

$\rho$ - flow density, $\mathrm{kg} \cdot \mathrm{m}^{-3}$;

$R e$ - Reynolds number;

$V$ - velocity of the flow in the channel, $\mathrm{m} \cdot \mathrm{s}^{-1}$.

The exponents $n$ and $m$ are selected depending on the flow characteristic and the plate surface: for turbulent flow (within $\operatorname{Re}=50 \ldots 30000$ and $\operatorname{Pr}=0.7 \ldots 80) n=0.73 ; m=0.43$; with laminar flow $(\operatorname{Re} \leq 50, \operatorname{Pr} \geq 80) n=m=0.33$. The coefficient $a$ is chosen depending on the surface area of the plate and the flow characteristic. Hydraulic resistance for each media $\Delta P(\mathrm{~Pa})$ :

$$
\Delta P=x \xi \frac{L n}{d_{e}} \cdot \frac{\rho \cdot V^{2}}{2}+3 \frac{\rho V_{n}^{2}}{2},
$$

where $x$-number of packages for specified media, connected in series, pcs;

$d_{e}$ - equivalent diameter of the channels, m;

$V, V_{n}$ - accordingly, velocity in the channels and in the inlet and outlet nozzles; if the fluid velocity in the nozzles is less than $2.5 \mathrm{~m} \cdot \mathrm{s}^{-1}$, their pressure drop can be ignored;

$\xi$ - coefficient of fluid resistance for the laminar flow (5):

$$
\xi=\frac{a_{1}}{R e}
$$

for the turbulent flow (6):

$$
\xi=\frac{a_{2}}{R e^{0.25}},
$$

where $a_{1}$ and $a_{2}$-coefficients chosen in accordance to the surface of the plate.

Evaluation of the heat transfer intensification effectivity coefficient proceeded in accordance to equation (7) [2]:

$$
E=\frac{N u / N u_{0}}{\left(\xi / \xi_{0}\right)^{1 / 3}},
$$

where $N u_{0}$ and $\xi_{0}$ - Nusselt number and hydraulic resistance coefficient for the base model NB538-V0.

\section{Results and discussion}

The results showed that, compared with the base variant, a change in the geometric parameters of the plate led to a change in both the Reynolds number and the Nusselt number, taking into account the fact that in all the considered cases the working parameters of heat exchangers are the same. Compared to the base NB538-V0, for the heat exchanger NB538-V4 the heat transfer coefficient $\mathrm{Nu}$ 
increased by $6 \%$. The graph of the Nusselt number $(\mathrm{Nu})$ versus the Reynolds number $(\mathrm{Re})$ for the options under consideration is shown in Figure 4.

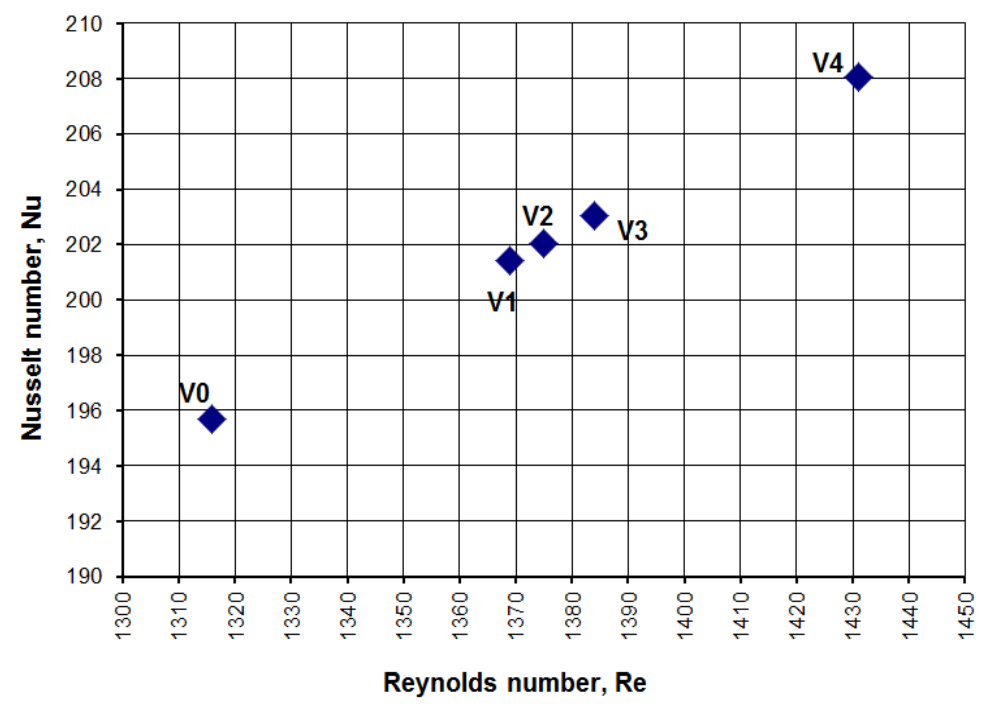

Fig. 4. Graph of Nusselt number $(\mathrm{Nu})$ versus Reynolds number $(\boldsymbol{R e})$ : V0 - base case NB538-V0, V1 - NB538-V1, V2 - NB538-V2, V3 - N538-V3, V4 - N38-V4

At the same time, the coefficient of fluid resistance $\xi$ for the heat exchanger NB538-V4, compared with the base version NB538-V0, fell by $9 \%$. A graph of the dependence of the coefficient of fluid resistance $\xi$ on the Reynolds number $(R e)$ for the considered variants is shown in Fig. 5.

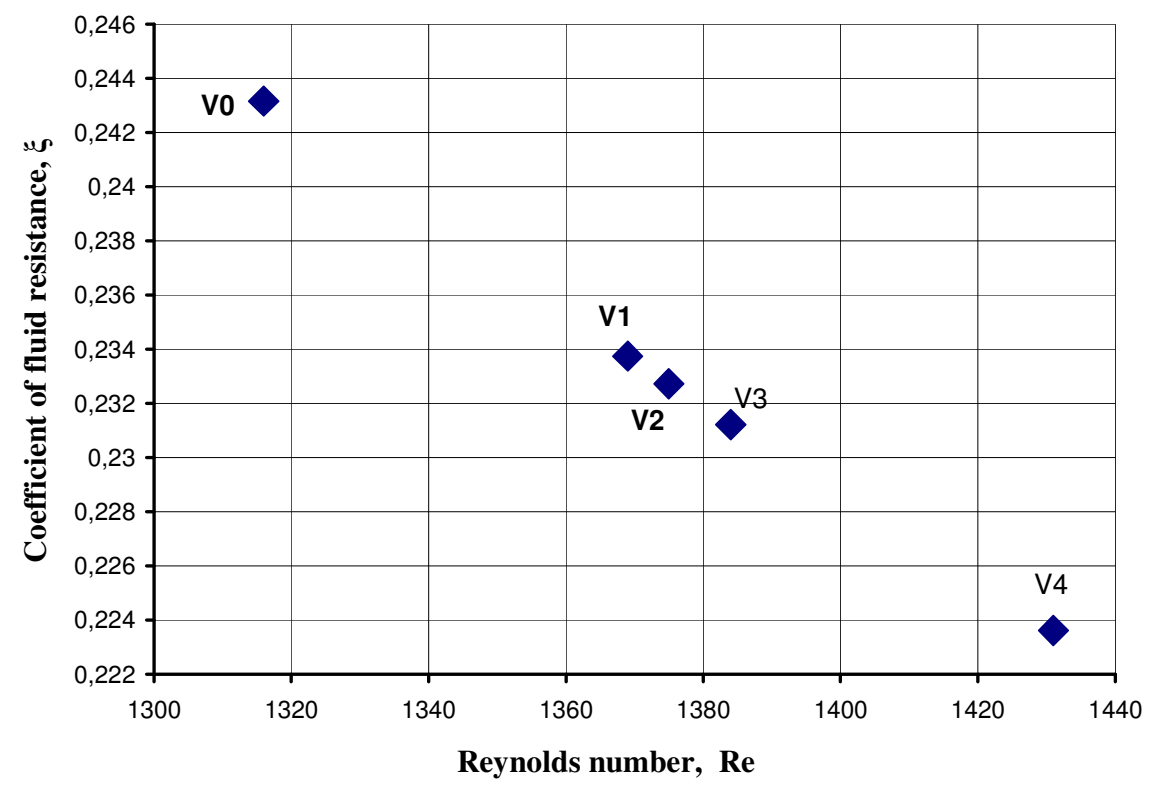

Fig. 5. Graph of dependence of coefficient of fluid resistance $\boldsymbol{\xi}$ on Reynolds's number $(\boldsymbol{R} \boldsymbol{e})$ : V0 - base case NB538-V0, V1 - NB538-V1, V2 - NB538-V2, V3 -N538-V3, V4 - N38-V4

Based on the obtained results a prototype of the tool and prototype of the heat exchanger with an optimized version of NB538-V4 with improved thermophysical properties were designed and manufactured.

The results of the evaluation of the heat transfer intensification effectivity coefficient for the considered variants of the heat exchangers showed that, compared with the basic variant NB538-V0, the increase in heat exchange for the variant V4-N38-V4 was $\approx 9 \%$. 


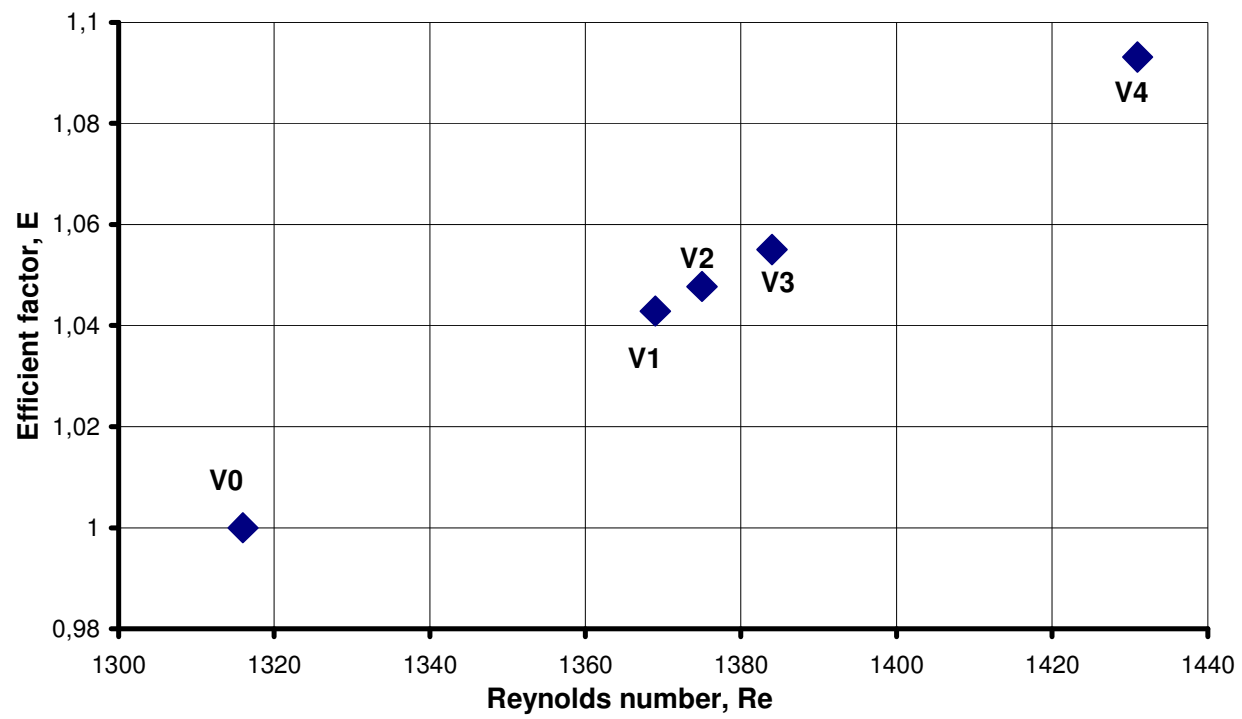

Fig. 6. Graph of dependence of heat transfer intensification effectivity coefficient $(E)$ on Reynolds's number $(\boldsymbol{R} e)$ : V0 - base case NB538-V0, V1 - NB538-V1, V2 -NB538-V2, $\mathrm{V} 3-\mathrm{N} 538-\mathrm{V} 3, \mathrm{~V} 4-\mathrm{N} 38-\mathrm{V} 4$.

\section{Conclusions}

1. The geometry of the heat exchanger plates was optimized, which took into account the peculiarities of the flow of the media in the channels for the brazed plate heat exchanger. Calculations of the thermo-physical and hydraulic parameters of the base and optimized heat transferring plates intended for the installation into the heat exchanger type NB538 with maximal operating pressure 31 bar and H-type of the plate are carried out.

2. The calculation results showed that for the optimized version of the NB538-V4 plates comparing with the base NB538-V0 the heat transfer coefficient $(\mathrm{Nu})$ increased by $6 \%$, with reducing of the hydraulic resistance $(\xi)$ by $9 \%$.

3. Comparing of the various models was carried out on the basis of heat removal from a plate's surface unit at equal specific powers required to overcome hydraulic losses. Evaluation of the efficiency of heat transfer intensification $(E)$ showed increasing in heat transfer $\approx 9 \%$. Thus, for the considered models, the increase in heat transfer is energetically greater in weight than the increase in pressure loss.

4. For further testing purposes for the strength and tightness of the final product the prototype of the plates was developed and manufactured, as well as a design of the prototype of the heat exchanger with optimized plate's shape NB538-V4 with improved thermal properties.

\section{Acknowledgements}

This paper has been published within the research project SIA "EKO AIR" study No. 2.2. "Increasing the strength of heat exchangers soldering joints" within the project "Establishment of the Centre of Mechanical Engineering Competence", for which LLC "MAŠĨNBŪVES KOMPETENCES CENTRS" and the Central Finance and Contracting Agency signed the contract No. 1.2.1.1/16/A/ on October 11, 2016 003, Project No 1.2.1.1/16/A/003.

\section{References}

[1] Danilov O.L., Garyaev A.B., Yakovlev I.V., Klimenko A.V., Vakulko A.G. Energy savings in power and heat technologies [electronic resource]: a textbook for high schools - Electron. Dan. M.: MEI Publishing House, 2010, 424 p.

[2] Klimenkov, Yu. V. V. A. Nekrashevich, A. E. Sinkevich Heat-hydraulic testing of the heat exchanging modules for heat and water supply systems, Energy efficiency. 2003, № 12, pp. 15-17.

[3] Migay V.K. Improving the efficiency of modern heat exchangers. L:Energiya 1980.144 p. 
[4] Compact Heat Exchangers for Energy Transfer Intensification: Low Grade Heat and Fouling Mitigation. / Klemes J.J., Arsenyeva O., Kapustenko P., Tovazhnyanskyy L.: CRC Press, 2015. $354 \mathrm{p}$.

[5] Anipko O.B., Arseneva O.P., Kapustenko P.A. Experimental equipment for thermal and hydraulic testing of plate heat exchangers. / Proceedings of the Odessa National Academy of Food Technologies. Odessa, ONAPT, 2003, № 25., pp. 74-79.

[6] Kulinichenko V.R. Handbook for the heat exchange calculations. Kiev. Tehnika. 1990, 165 p.

[7] Kapustenko P.O., Kukulka D.J., Arsenyeva O.P. Intensification of Heat Transfer Processes // Chemical Engineering Transactions. - 2015.-T. 45. pp. 1729-1734.

[8] Chang P. Separation of Flow //New York, Pergamon Press, 1971.

[9] Miheev M.A. Miheeva I.M. Basics of the heat transfer. - M.: Energiya. 1977, 344 p. 